\title{
Fully automated, inline quantification of myocardial blood flow with cardiovascular magnetic resonance: repeatability of measurements in healthy subjects
}

Louise A. E. Brown', Sebastian C. Onciul', David A. Broadbent ${ }^{1,2}$, Kerryanne Johnson', Graham J. Fent', James R. J. Foley ${ }^{1}$, Pankaj Garg ${ }^{1}$, Pei G. Chew ${ }^{1}$, Kristopher Knott ${ }^{3}$, Erica Dall'Armellina ${ }^{1}$, Peter P. Swoboda' ${ }^{1}$ Hui Xue ${ }^{4}$, John P. Greenwood', James C. Moon ${ }^{3}$, Peter Kellman ${ }^{4}$ and Sven Plein ${ }^{1 *}$

\begin{abstract}
Background: Non-invasive assessment of myocardial ischaemia is a cornerstone of the diagnosis of coronary artery disease. Measurement of myocardial blood flow (MBF) using positron emission tomography (PET) is the current reference standard for non-invasive quantification of myocardial ischaemia. Dynamic myocardial perfusion cardiovascular magnetic resonance (CMR) offers an alternative to PET and a recently developed method with automated inline perfusion mapping has shown good correlation of MBF values between CMR and PET. This study assessed the repeatability of myocardial perfusion mapping by CMR in healthy subjects.

Methods: Forty-two healthy subjects were recruited and underwent adenosine stress and rest perfusion CMR on two visits. Scans were repeated with a minimum interval of 7 days. Intrastudy rest and stress MBF repeatability were assessed with a 15-min interval between acquisitions. Interstudy rest and stress MBF and myocardial perfusion reserve (MPR) were measured for global myocardium and regionally for coronary territories and slices.

Results: There was no significant difference in intrastudy repeated global rest MBF $(0.65 \pm 0.13 \mathrm{ml} / \mathrm{g} / \mathrm{min}$ vs $0.62 \pm 0.12 \mathrm{ml} / \mathrm{g} / \mathrm{min}, p=0.24$, repeatability coefficient $(\mathrm{RC})=24 \%)$ or stress $(2.89 \pm 0.56 \mathrm{ml} / \mathrm{g} / \mathrm{min}$ vs $2.83 \pm 0.64 \mathrm{ml} / \mathrm{g} / \mathrm{min}$, $p=0.41, \mathrm{RC}=29 \%) \mathrm{MBF}$. No significant difference was seen in interstudy repeatability for global rest MBF $(0.64 \pm 0.13 \mathrm{ml} /$ $\mathrm{g} / \mathrm{min}$ vs $0.64 \pm 0.15 \mathrm{ml} / \mathrm{g} / \mathrm{min}, p=0.80, \mathrm{RC}=32 \%)$, stress MBF $(2.71 \pm 0.61 \mathrm{ml} / \mathrm{g} / \mathrm{min}$ vs $2.55 \pm 0.57 \mathrm{ml} / \mathrm{g} / \mathrm{min}, p=0.12$, $\mathrm{RC}=33 \%)$ or MPR ( $4.24 \pm 0.69$ vs $3.73 \pm 0.76, p=0.25, \mathrm{RC}=36 \%)$. Regional repeatability was good for stress $(\mathrm{RC}=30-37 \%)$ and rest $M B F(R C=32-36 \%)$ but poorer for $M P R(R C=35-43 \%)$. Within subject coefficient of variation was $8 \%$ for rest and $11 \%$ for stress within the same study, and $11 \%$ for rest and $12 \%$ for stress between studies.
\end{abstract}

Conclusions: Fully automated, inline, myocardial perfusion mapping by CMR shows good repeatability that is similar to the published PET literature. Both rest and stress MBF show better repeatability than MPR, particularly in regional analysis.

Keywords: Myocardial perfusion, Cardiac magnetic resonance, Myocardial perfusion reserve

\footnotetext{
* Correspondence: s.plein@leeds.ac.uk

Dr. Eike Nagel served as a Guest Editor for this manuscript.

${ }^{1}$ Multidisciplinary Cardiovascular Research Centre (MCRC) \& Leeds Institute of

Cardiovascular and Metabolic Medicine, University of Leeds, Clarendon Way,

Leeds LS2 9JT, UK

Full list of author information is available at the end of the article
}

(c) The Author(s). 2018 Open Access This article is distributed under the terms of the Creative Commons Attribution 4.0 International License (http://creativecommons.org/licenses/by/4.0/), which permits unrestricted use, distribution, and reproduction in any medium, provided you give appropriate credit to the original author(s) and the source, provide a link to the Creative Commons license, and indicate if changes were made. The Creative Commons Public Domain Dedication waiver (http://creativecommons.org/publicdomain/zero/1.0/) applies to the data made available in this article, unless otherwise stated. 


\section{Background}

There is increasing evidence that revascularisation decisions in patients with coronary artery disease (CAD) should be based on objective measurements of ischaemia rather than visual assessment [1-3]. Positron emission tomography (PET) is the current reference standard for non-invasive quantification of myocardial blood flow (MBF) and detection of ischaemia. Quantitative perfusion cardiovascular magnetic resonance (CMR) offers an alternative to PET with the advantage of lack of ionising radiation, higher spatial resolution and the added value of providing comprehensive data on left ventricular size, function and scar within a single study. Quantification of MBF by CMR has been validated in several small scale studies against microspheres, PET and invasive fractional flow reserve (FFR) measurements [4-7]. However, the wider adoption of quantitative perfusion CMR has been limited by the need for time consuming, off line processing and poor repeatability [8]. Recently, a new motion corrected (MOCO) myocardial perfusion CMR method with Gadgetron-based automated in-line perfusion mapping has been proposed, allowing free breathing acquisition and pixel-wise quantification of MBF [9]. The method has been shown to have good correlation with PET in patients with stable CAD [10], but needs to proceed through a number of validation steps before it can be used clinically and as a research surrogate endpoint. In this study, the repeatability of myocardial perfusion mapping by CMR was assessed in healthy subjects.

\section{Methods}

\section{Study population}

Forty-two healthy subjects were recruited (23 female, median age 23 yrs., IQR 22-29 yrs). Exclusion criteria were known cardiovascular disease, hypertension, hyperlipidaemia, diabetes mellitus, smoking and any contraindication to $\mathrm{CMR}$, adenosine or gadolinium based contrast agents.

\section{Study protocol}

The 42 healthy subjects underwent repeat CMR studies in two groups to allow assessment of intrastudy and interstudy repeatability of rest and stress MBF as well as MPR.

a) Group 1: 30 subjects underwent CMR studies on two separate visits. In visit 1 , they had three rest perfusion scans and in visit 2 , they had two stress and one rest perfusion scans. The acquisition of three rest scans in visit 1 allowed us to study the short-term repeatability of rest MBF with minimal physiological variation and to assess the effect of residual gadolinium $(\mathrm{Gd})$ on repeated perfusion measurements. The fourth rest scan acquired in visit 2 was used to assess long-term variability of rest MBF.
The two stress scans in visit 2 assessed short-term variability of stress $\mathrm{MBF}$.

b) Group 2: 20 subjects (8 from the first cohort and 12 additional subjects) had stress followed by rest perfusion scans in two separate visits to assess longterm repeatability of stress MBF and MPR (Fig. 1).

All CMR studies were carried out on a $3 \mathrm{~T}$ scanner (Magnetom Prisma, Siemens Healthineers, Erlangen, Germany). A minimum of 7 days was allowed between each visit (mean 41, SD 40 days). Subjects were advised to avoid caffeine for $24 \mathrm{~h}$ before each scan. Survey images were acquired, followed by vertical and horizontal long axis images to plan the short axis view for perfusion in three slice positions (basal, mid and apex).

Pharmacological stress was achieved with adenosine infusion at $140 \mathrm{mcg} / \mathrm{kg} / \mathrm{min}$ for a minimum of $3 \mathrm{~min}$. The dose was increased to $175 \mathrm{mcg} / \mathrm{kg} / \mathrm{min}$ after $2 \mathrm{~min}$ if there was no symptomatic or haemodynamic response to adenosine. Subjects were monitored for symptoms and heart rate throughout the infusion, blood pressure and heart rate were recorded every 2 min during adenosine infusion. An intravenous bolus of $0.05 \mathrm{mmol} / \mathrm{kg}$ gadobutrol (Gadovist, Leverkusen, Germany) was administered at $5 \mathrm{ml} / \mathrm{s}$ followed by a $20 \mathrm{ml}$ saline flush for each perfusion scan. A minimum gap of 15 min was maintained between each perfusion scan to allow for equilibrium of gadolinium

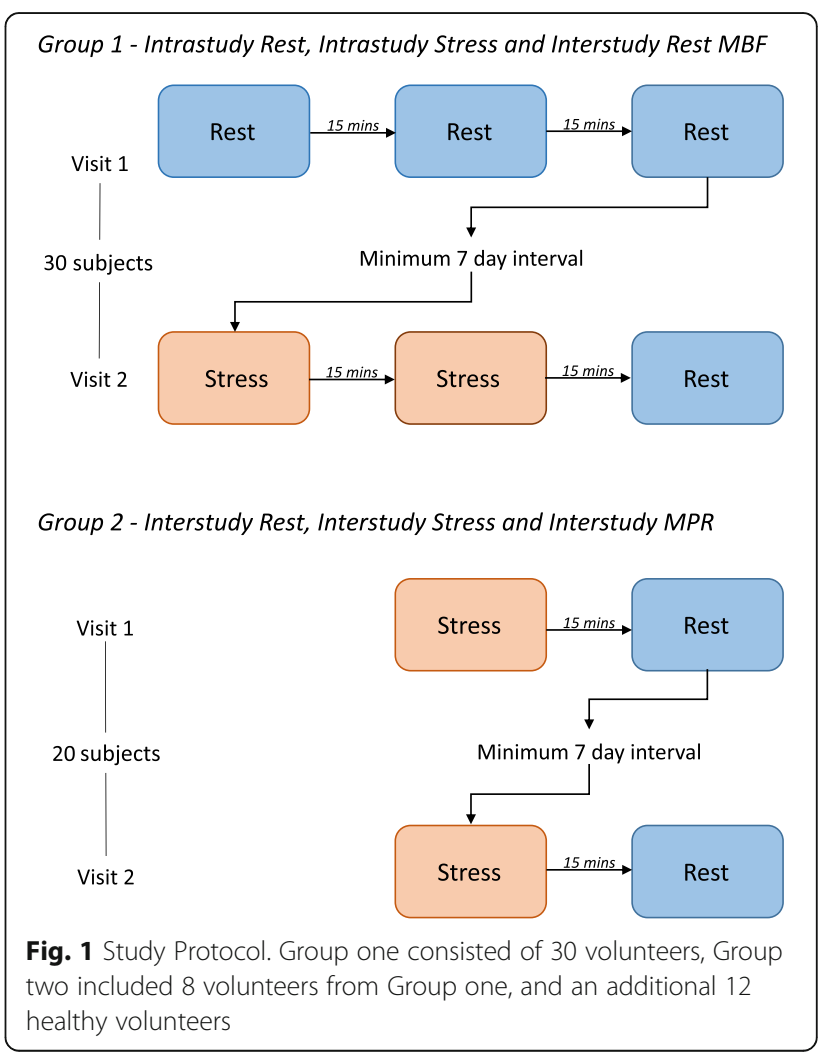


kinetics from the previous series, and to ensure that the effects of adenosine had resolved.

Perfusion imaging used a dual sequence approach which employed separately optimized sequences for the myocardium and blood pool signals in order to avoid blood pool signal saturation. Full details of the myocardial perfusion sequence have been previously described [9]. Both sequences were electrocardiogram (ECG) triggered saturation recovery prepared. The sequence used for imaging the left ventricular (LV) blood pool to estimate the arterial input function (AIF) used a low flip angle FLASH low resolution protocol with 2 echos such that the echo times were short to minimize T2* losses at high concentration, and so that remaining $\mathrm{T} 2 *$ losses could be estimated and corrected. Parameters for this protocol were: flip angle 5 degrees, matrix $48 \times 34$, parallel imaging factor 2, TEs 0.76 and $1.76 \mathrm{~ms}$, TR $2.45 \mathrm{~ms}$, slice thickness $10 \mathrm{~mm}$, saturation preparation used 6-pulse sequence, saturation delay TS $24 \mathrm{~ms}$ to $\mathrm{k}$-space center, imaging duration $42 \mathrm{~ms}$, total sequence duration $57 \mathrm{~ms}$ acquired immediately following the R-wave trigger. The myocardial imaging protocol in this study used a FLASH readout with typical imaging parameters: flip angle 14 degrees, spatial resolution $1.9 \times 2.4 \mathrm{~mm} 2$, slice thickness $8.0 \mathrm{~mm}$, TE/TR 1.0/2.1 ms, matrix size $192 \times$ 111, field of view $360 \times 270 \mathrm{~mm} 2$, parallel imaging acceleration factor 3, saturation recovery time (TS) $110 \mathrm{~ms}$ to center of k-space, trigger delay $72 \mathrm{~ms}$, imaging duration $59 \mathrm{~ms}$, saturation preparation used 5-pulse sequence, total duration including saturation $143 \mathrm{~ms}$ per slice, enabling acquisition of 3 -slices plus AIF sequence in less than $500 \mathrm{~ms}$ permitting hear rates up to $120 \mathrm{bpm}$. Both AIF and myocardial imaging sequences included 3 measurements of proton density (PD) weighted images with flip angle of 5 degrees used for surface coil intensity correction. Slice spacing was varied on per patient basis to cover the LV.

In-line automatic reconstruction and post-processing were implemented within the Gadgetron software framework [9]. Images were motion corrected and then corrected for surface coil intensity variation based on the proton density weighted images. Signal intensity data were converted to $\mathrm{Gd}$ concentration $(\mathrm{mmol} / \mathrm{L})$ based on automatically generated look-up tables for the magnetization Bloch simulation. AIF data were extracted from the low-resolution Gd concentration images using automated segmentation of the LV cavity. MBF was calculated on a pixel-wise basis in the high-resolution images by blood tissue exchange (BTEX) model constrained deconvolution incorporating estimation of the delay time between bolus arrival in the LV cavity and the tissue of interest. Details of the reconstruction and processing including conversion to [Gd] concentration units, blood pool signal segmentation, and BTEX modeling are previously reported [9].

\section{Quantitative analysis}

The in-line processing on the scanner console included the image reconstruction, respiratory motion correction, LV blood pool segmentation, conversion of signals to [Gd] concentration units, and quantitative tissue mapping. These steps were fully automatic. For a protocol with AIF plus 3-slices acquired for 60 measurements, the processing time was $<3$ min.

The analysis of the quantitative maps was performed off-line on a separate workstation using CVi 42 software (Circle Cardiovascular Imaging, Calgary, Canada). This process was performed manually by tracing endo- and epi-cardial contours for each slice and marking RV insertion points; a 16-segment American Heart Association (AHA) model was then applied [11]. In order to minimise partial volume effect, a $10 \%$ offset was applied to endocardial and epicardial borders (Fig. 2). MBF was recorded for each of the 16 segments. Where the LV outflow tract was included in the basal slice, or partial volume effect meant segments were too thin to contour, these segments were excluded from further analysis. Analysis time was less than 5 min per set of perfusion maps.

Segmental values were averaged to give values for slice, coronary territory and global MBF. Coronary territories consisted of: left anterior descending coronary artery (LAD) - segments 1, 2, 7, 8, 13 and 14, circumflex coronary artery $(\mathrm{Cx})$ - segments 5, 6, 11, 12 and 16, and right coronary artery (RCA) - segments 3, 4, 9, 10 and 15.

Correlation of MBF with HR and RPP were analysed, and where significant correlation was present, MBF values were corrected. Values for resting MBF were corrected for heart rate (HR) by dividing by scan heart rate and multiplying by the mean resting HR (62 bpm) among all subjects. Interstudy repeatability was analysed on a regional basis for slices (basal, mid and apical) and coronary territories (LAD, Cx and RCA). MPR was calculated as a ratio of stress MBF:rest MBF.

\section{Reproducibility of analysis}

Intra- and inter-observer variability were assessed by repeating the analysis of 10 subject data sets for stress and rest after 1 month, by the same observer (LB) and by a second observer (SO). The second observer was blinded to the previous results.

\section{Statistical analysis}

Analysis was performed using SPSS 23 (International Business Machines, Armonk, New York, USA). Normality of data distribution was assessed using Shapiro-Wilk test. Data are presented as mean \pm standard deviation (SD). Repeatability was assessed using a wide range of methods to facilitate comparison with the inconsistent methods in the published literature. The three intrastudy rest scans were compared using repeated measures analysis of variance 

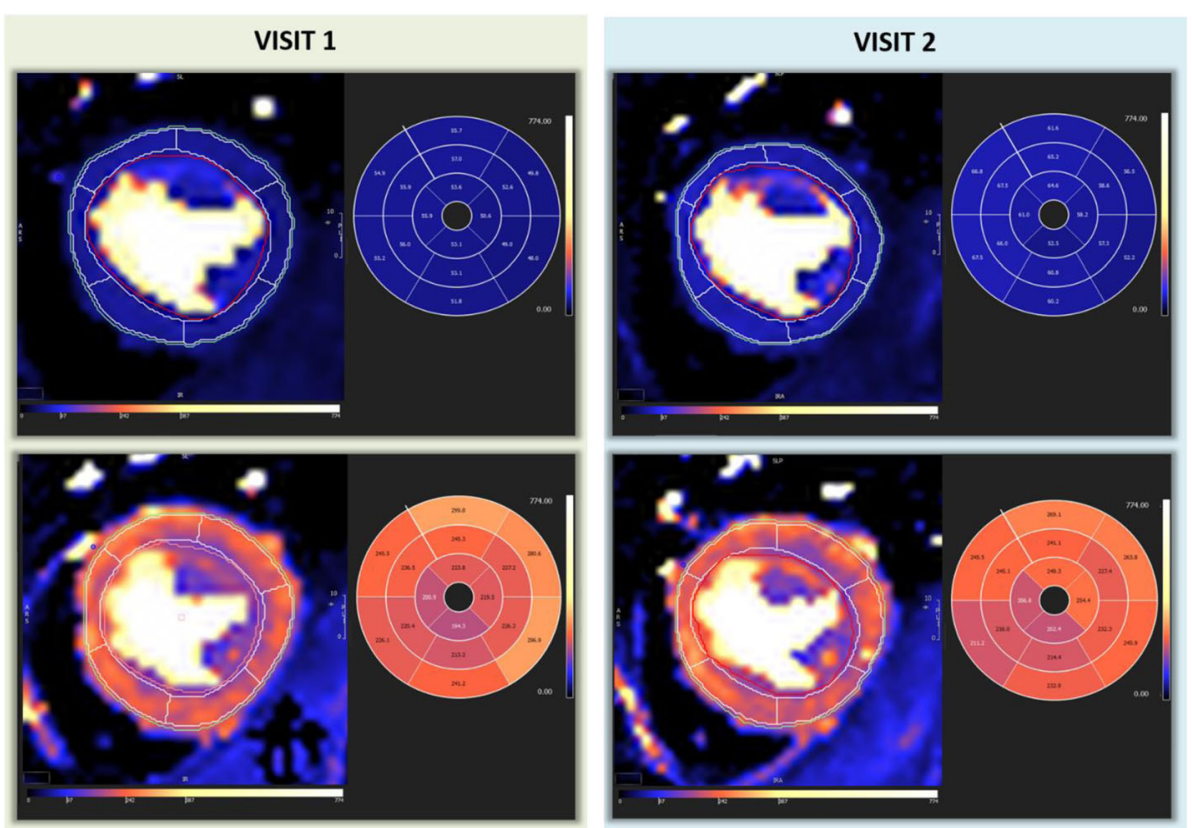

Fig. 2 Rest and Stress MBF maps from visit 1 and visit 2 for the same subject. Values are displayed as ml/100 g/min

(ANOVA) with Bonferroni adjustment for post-hoc analysis. All other repeated mean MBF and inter- and intraobserver variability were compared using paired $t$ tests. Coefficient of variation $(\mathrm{CV})$ was calculated using the root mean square method [12]. Reproducibility coefficient (RC) was calculated as $1.96^{*} \mathrm{SD}$ of difference and given as a percentage of the total mean and used to demonstrate bias and accuracy with Bland Altman plots. Reliability was assessed using intraclass correlation coefficient (ICC). All statistical tests were two-tailed and a $p$ value $<0.05$ was considered significant.

\section{Results}

All subjects tolerated repeated CMR scans and adenosine stress well. One subject did not attend the second visit for assessment of intrastudy rest repeatability, another was not included in intrastudy stress analysis due to triggering problems causing artefact on the MBF maps on one stress scan. One result was excluded from analysis of repeat stress MBF or MPR due to lack of stress response on one visit, confirmed by lack of symptoms despite increased adenosine dose, haemodynamic response and splenic switch off. One result was excluded from comparison of MPR due to severe artefact on rest perfusion maps.

\section{Intrastudy repeatability}

Twenty-nine studies were analysed for intrastudy repeatability of resting and hyperaemic MBF from the two separate visits of Group 1.

Mean global MBF at rest was $0.69 \pm 0.13 \mathrm{ml} / \mathrm{g} / \mathrm{min}, 0.65 \pm$ $0.13 \mathrm{ml} / \mathrm{g} / \mathrm{min}$ and $0.62 \pm 0.12 \mathrm{ml} / \mathrm{g} / \mathrm{min}$ for scans 1,2 and 3 respectively (Table 1 ). There was a significant difference in mean MBF on the first rest scan compared to both the second $(p=0.01)$ and third $(p=0.001)$. There was no

Table 1 Intrastudy repeatability of global MBF measurements

\begin{tabular}{|c|c|c|c|c|c|c|c|c|c|c|c|}
\hline & \multicolumn{2}{|c|}{$\begin{array}{l}\text { Test } 1 \\
\mathrm{ml} / \mathrm{g} / \mathrm{min}\end{array}$} & \multicolumn{2}{|c|}{ Test $2 \mathrm{ml} / \mathrm{g} / \mathrm{min}$} & \multicolumn{2}{|c|}{$\begin{array}{l}\text { Difference in } \\
\text { mean } \mathrm{ml} / \mathrm{g} / \mathrm{min}\end{array}$} & $p$ & $\mathrm{RC}$ & $\mathrm{RC}(\%)$ & CV (\%) & ICC \\
\hline \multicolumn{7}{|l|}{ Rest } & \multicolumn{5}{|l|}{$<0.01$} \\
\hline Rest 1 - Rest 2 & 0.69 & \pm 0.13 & 0.65 & \pm 0.13 & -0.04 & \pm 0.09 & 0.04 & 0.19 & 28.5 & 10.9 & 0.73 \\
\hline Rest 1 - Rest 3 & 0.69 & \pm 0.13 & 0.62 & \pm 0.12 & -0.07 & \pm 0.10 & 0.02 & 0.23 & 35.2 & 11.9 & 0.58 \\
\hline Rest 2 - Rest 3 & 0.65 & \pm 0.13 & 0.62 & \pm 0.12 & -0.03 & \pm 0.07 & 0.24 & 0.15 & 23.8 & 7.93 & 0.80 \\
\hline \multicolumn{12}{|l|}{ Stress } \\
\hline Stress 1- Stress 2 & 2.89 & \pm 0.56 & 2.83 & \pm 0.64 & -0.06 & \pm 0.42 & 0.41 & 0.82 & 28.5 & 10.6 & 0.76 \\
\hline
\end{tabular}

$p$ - from repeated measures ANOVA and level of significance using Bonferroni post-hoc analysis for rest data, Student's T-test for stress values $R C$ repeatability coefficient, $R C$ (\%)repeatability coefficient as percentage of the mean, $C V$ within subject coefficient of variation, ICC intraclass correlation coefficient 
significant difference between the second and third scans (Fig. 3). Coefficient of variation was $11-12 \%$ between the first scan and repeats, and $8 \%$ between second and third scans with good reliability (ICC $=0.8, \mathrm{RC} 24 \%$ ). Assessment of repeatability with Bland-Altman plots (Fig. 4) showed a bias of $-0.03 \mathrm{ml} / \mathrm{g} / \mathrm{min}$ (3.9\% of the mean).

Resting MBF correlated with HR $(r=0.49, p<0.01)$, therefore MBF corrected for $\mathrm{HR}$ was also analysed (Table 2). A significant difference was still present between the groups $(p<0.01)$ and the decrease with sequential scans remained $(0.69 \mathrm{ml} / \mathrm{g} / \mathrm{min}, 0.66 \mathrm{ml} / \mathrm{g} / \mathrm{min}$ and $0.61 \mathrm{ml} / \mathrm{g} / \mathrm{min}$ for scans 1,2 and 3). A significant difference was seen between scan 3 and the other two results $(p<0.01)$. Whilst the level of significance differed when corrected for heart rate, both sets of values showed a trend to decrease with repeated measurement (Fig. 3).

Stress MBF showed no significant difference between the two repeat acquisitions in visit 2 (mean difference $-0.06 \pm$ $0.42, p=0.41)$. Within subject coefficient of variation was
$11 \%$ with good correlation and repeatability (ICC 0.76, RC $29 \%$ ). One value was outside the limits of agreement on assessment with Bland-Altman plots, with a bias of $2.2 \%$ of the mean (Fig. 4). Stress RPP was comparable between both studies $(11,202 \pm 2188$ vs $10,858 \pm 1877, p=0.09)$ as was the percentage increase in HR $(47.3 \pm 18.8 \%$ vs $44.4 \pm 18.4 \%, p=$ $0.24)$ and RPP ( $51.1 \pm 21.7 \%$ vs $46.9 \pm 22.1 \%, p=0.14)$.

\section{Interstudy repeatability \\ Global perfusion analysis}

A total of 41 studies were analysed for interstudy repeatability of resting MBF with an average gap of 27 days between scans in visits 1 and 2 . No significant difference was seen in MBF between scans (mean difference $0.004 \pm 0.1 \mathrm{ml} / \mathrm{g} / \mathrm{min}$, $p=0.8$ ) (Table 3). Within subject coefficient of variation was $11 \%$, RC $32 \%$ and bias was $<1 \%$ of the mean (Fig. 5).

Nineteen pairs of scans were analysed for interstudy stress MBF and 18 for interstudy MPR in Group 2. The percentage increase in HR $(52.1 \pm 26.6 \%$ vs $50.4 \pm 23.4 \%, p=0.7)$ and

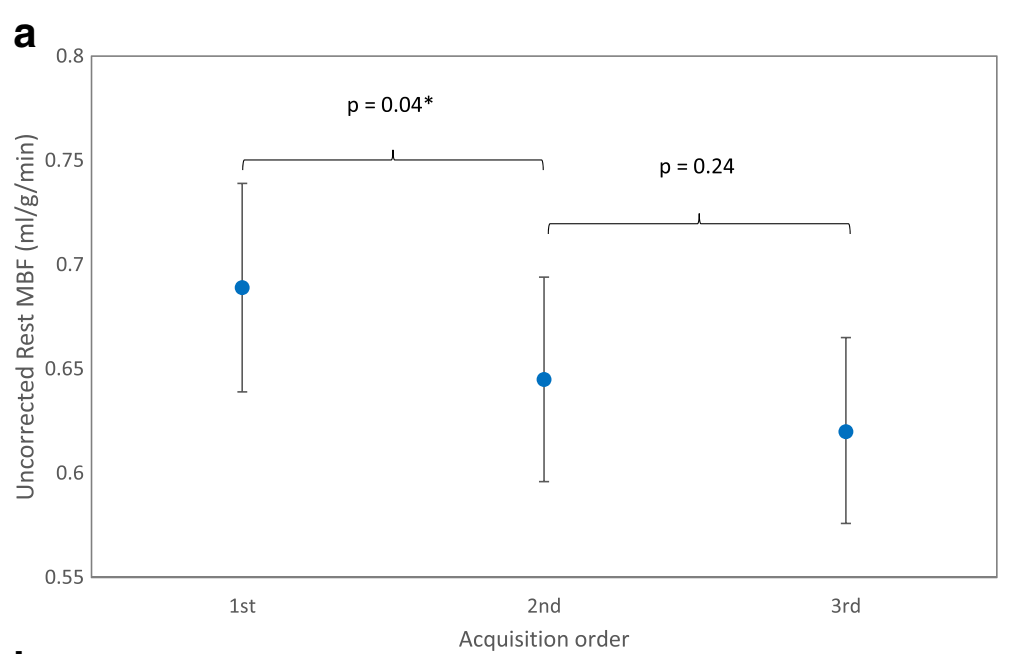

b

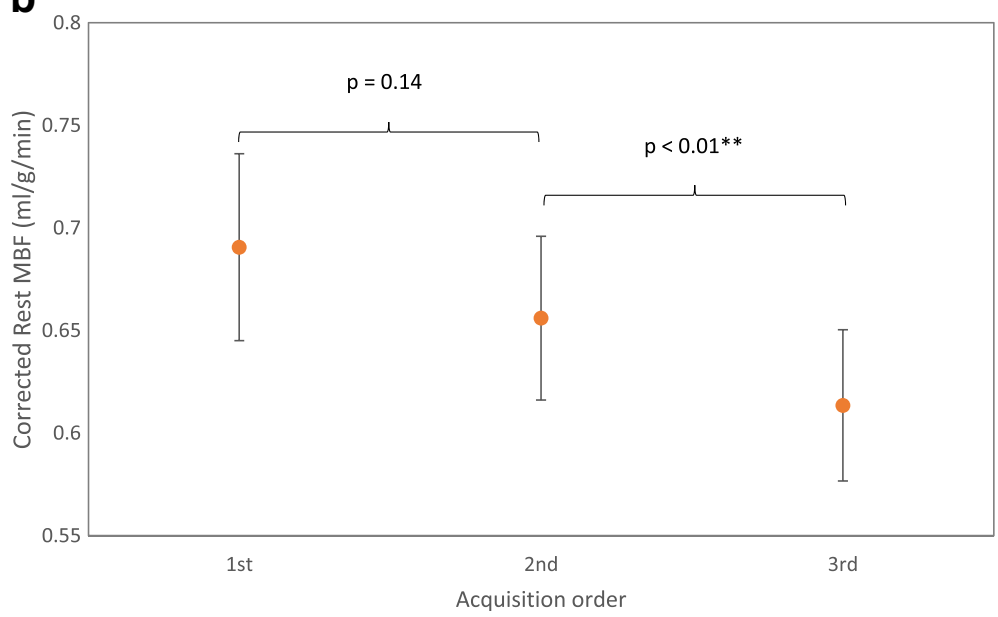

Fig. 3 Global resting myocardial blood flow (MBF) on repeat throughout the same visit. A - uncorrected values, B - corrected values (mean and $95 \%$ confidence interval of the mean) 


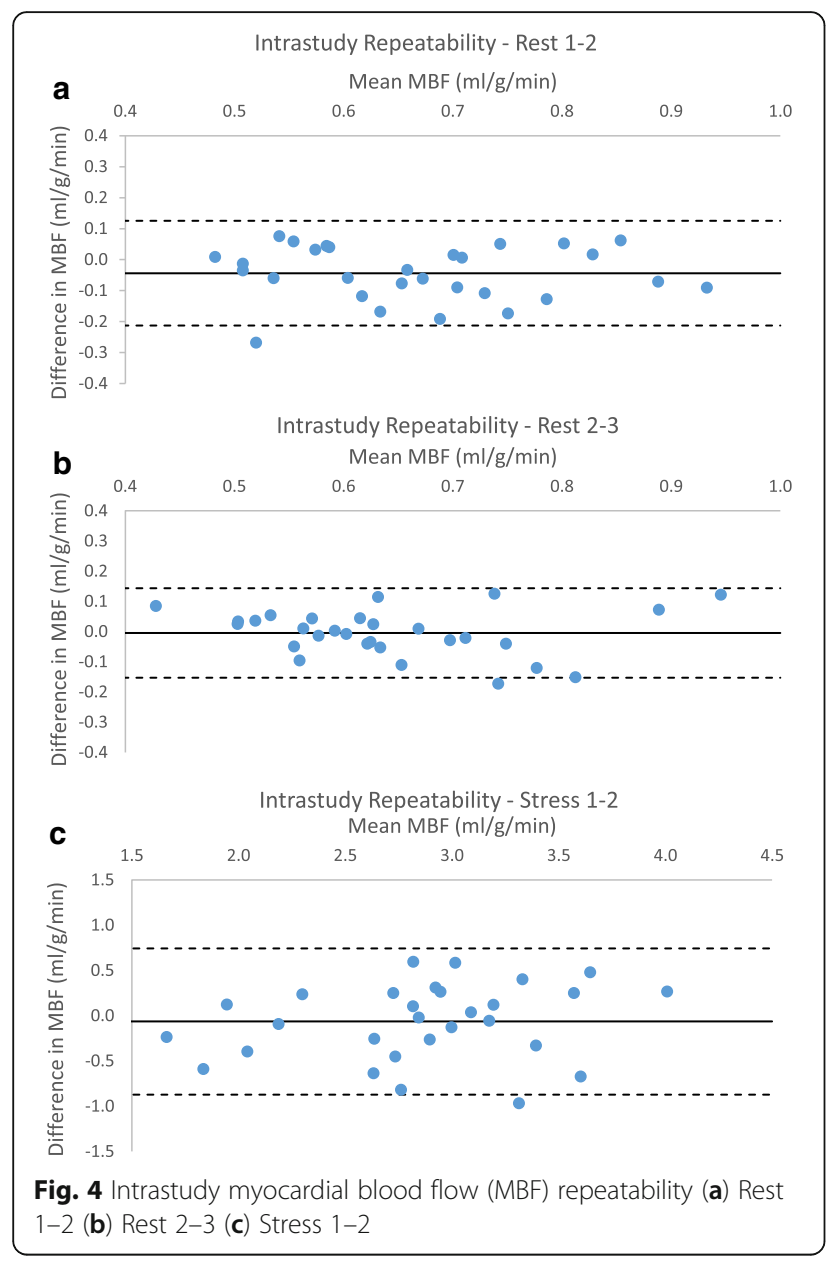

RPP $(56.6 \pm 32.7 \%$ vs $52.1 \pm 26.6 \%, p=0.11)$ between rest and stress scans showed no significant difference between visits. Adenosine stress $\mathrm{MBF}$ had good repeatability with ICC 0.72 and RC 33\%. CV was $12 \%$ and bias was $-6 \%$ of the mean. Repeat MPR had a CV of 13.3 with no significant difference between the two measurements. Weaker correlation was seen compared to stress and rest, although this remained significant (ICC 0.46, $p<0.01$ ).

\section{Regional perfusion analysis}

At rest there was no significant difference between slices ( $p=0.13$ and 0.09 for first and second scans) (Table 3). No significant difference was seen in individual slices between scans and all showed good repeatability and correlation $(p<0.01$ in all slices) (Additional file 1: Figure S1). Coefficient of variation was 13.6, 11.2 and $11.5 \%$ for basal, mid and apical slices respectively.

Mean MBF at stress was $3.01 \pm 0.8 \mathrm{ml} / \mathrm{g} / \mathrm{min}, 2.48 \pm$ $0.55 \mathrm{ml} / \mathrm{g} / \mathrm{min}$ and $2.62 \pm 0.65 \mathrm{ml} / \mathrm{g} / \mathrm{min}$ on the first visit and $2.8 \pm 0.74 \mathrm{ml} / \mathrm{g} / \mathrm{min}, 2.39 \pm 0.51 \mathrm{ml} / \mathrm{g} / \mathrm{min}$ and 2.42 $\pm 0.56 \mathrm{ml} / \mathrm{g} / \mathrm{min}$ in basal, mid and apical slices respectively. No significant difference was seen between scans for any slice. The apical slice exhibited the lowest repeatability, ICC 0.46 , RC $49 \%$ and within subject coefficient of variation $16 \%$. Good correlation was seen in all slices (Additional file 1: Figure S1).

No significant difference was seen in mean MPR between visits for any slice, RCs were 40, 42 and 48\% and CVs 15, 15 and 16\% for basal mid and apical slices.

Coronary territory flows were significantly different between vessel territories at rest on both visits. MBF in the LAD was higher than the Cx territory on both visits (mean difference $0.09 \mathrm{ml} / \mathrm{g} / \mathrm{min}, p=0.01$ on the first scan, and $0.08 \mathrm{ml} / \mathrm{g} / \mathrm{min}, p=0.04$ on the second). There was good correlation within all coronary territories between scans (Additional file 2: Figure S2). All territories showed similar ICC (0.66-0.75) and repeatability coefficients (32-36\%). $\mathrm{CVs}$ were very similar between territories $(11.7,11.8$ and $12.6 \%)$.

No significant difference was seen between coronary territories in stress MBF, or in calculated MPR at either scan. All coronary territories showed good repeatability and correlation between scans. Coefficients of variation ranged between 10.8 and $15.4 \%$, being highest in the circumflex territory.

No significant difference was seen in MPR in any coronary territory between visits. The LAD and Cx territories showed acceptable correlation and repeatability coefficients (LAD: ICC 0.5, RC 35\%, Cx: ICC 0.54, RC $41 \%)$. The RCA territory did not show significant correlation between visits, $\mathrm{ICC}=0.23$.

\section{Interobserver and Intraobserver repeatability}

Ten sets of perfusion maps were assessed for intraobserver variability at a minimum gap of 4 weeks between analysis, and for interobserver variability. There was

Table 2 Global rest myocardial blood flow (MBF) corrected for heart rate (HR)

\begin{tabular}{|c|c|c|c|c|c|c|c|c|c|c|c|}
\hline & \multicolumn{2}{|c|}{ Test $1 \mathrm{ml} / \mathrm{g} / \mathrm{min}$} & \multicolumn{2}{|c|}{ Test $2 \mathrm{ml} / \mathrm{g} / \mathrm{min}$} & \multicolumn{2}{|c|}{ Difference in mean $\mathrm{ml} / \mathrm{g} / \mathrm{min}$} & \multirow{2}{*}{$\begin{array}{l}p \\
<0.01\end{array}$} & \multirow[t]{2}{*}{$\mathrm{RC}$} & \multirow[t]{2}{*}{ RC (\%) } & \multirow[t]{2}{*}{ CV (\%) } & \multirow[t]{2}{*}{ ICC } \\
\hline & & & & & & & & & & & \\
\hline Rest 1 - Rest 2 & 0.69 & \pm 0.12 & 0.66 & \pm 0.10 & -0.03 & \pm 0.09 & 0.14 & 0.18 & 27.3 & 9.9 & 0.66 \\
\hline Rest 1 - Rest 3 & 0.69 & \pm 0.12 & 0.61 & \pm 0.10 & -0.07 & \pm 0.07 & $<0.01$ & 0.20 & 30.3 & 10.5 & 0.62 \\
\hline Rest 2 - Rest 3 & 0.66 & \pm 0.10 & 0.61 & \pm 0.10 & -0.04 & \pm 0.06 & $<0.01$ & 0.15 & 23.7 & 8.3 & 0.72 \\
\hline
\end{tabular}

$\mathrm{p}$ - from repeated measures ANOVA and Bonferroni post-hoc analysis

$R C$ repeatability coefficient, $R C$ (\%)repeatability coefficient as percentage of the mean, $C V$ within subject coefficient of variation, ICC intraclass correlation coefficient 
Table 3 Interstudy repeatability of MBF measurements - by slice and coronary artery territory

\begin{tabular}{|c|c|c|c|c|c|c|c|c|c|c|c|}
\hline \multirow{2}{*}{$\overline{\text { Rest }}$} & \multicolumn{2}{|c|}{$\begin{array}{l}\text { Test } 1 \\
\mathrm{ml} / \mathrm{g} / \mathrm{min}\end{array}$} & \multicolumn{2}{|c|}{$\begin{array}{l}\text { Test } 2 \\
\mathrm{ml} / \mathrm{g} / \mathrm{min}\end{array}$} & \multicolumn{2}{|c|}{$\begin{array}{l}\text { Difference in } \\
\text { mean } \mathrm{ml} / \mathrm{g} / \mathrm{min}\end{array}$} & \multirow[t]{2}{*}{$p$} & \multirow[t]{2}{*}{$\mathrm{RC}$} & \multirow[t]{2}{*}{ RC (\%) } & \multirow[t]{2}{*}{ CV (\%) } & \multirow[t]{2}{*}{ ICC } \\
\hline & & & & & & & & & & & \\
\hline Global & 0.64 & \pm 0.13 & 0.64 & \pm 0.15 & 0.004 & \pm 0.10 & 0.8 & 0.20 & 31.5 & 11.3 & 0.74 \\
\hline Basal & 0.66 & \pm 0.14 & 0.67 & \pm 0.16 & 0.015 & \pm 0.13 & 0.46 & 0.25 & 37.7 & 13.6 & 0.65 \\
\hline Mid & 0.64 & \pm 0.14 & 0.64 & \pm 0.15 & -0.003 & \pm 0.10 & 0.87 & 0.20 & 31.0 & 11.2 & 0.76 \\
\hline Apex & 0.60 & \pm 0.13 & 0.60 & \pm 0.15 & -0.003 & \pm 0.10 & 0.86 & 0.20 & 33.4 & 11.5 & 0.73 \\
\hline P & 0.13 & & 0.089 & & & & & & & & \\
\hline LAD & 0.69 & \pm 0.16 & 0.68 & \pm 0.16 & -0.003 & \pm 0.11 & 0.86 & 0.22 & 32.2 & 11.7 & 0.75 \\
\hline$C x$ & 0.60 & \pm 0.13 & 0.60 & \pm 0.14 & 0.005 & \pm 0.11 & 0.74 & 0.20 & 34.0 & 11.8 & 0.70 \\
\hline RCA & 0.61 & \pm 0.12 & 0.62 & \pm 0.15 & 0.01 & \pm 0.11 & 0.59 & 0.22 & 35.6 & 12.6 & 0.66 \\
\hline P & $0.01^{*}$ & & $0.04^{*}$ & & & & & & & & \\
\hline \multicolumn{12}{|l|}{ Stress } \\
\hline Global & 2.71 & \pm 0.61 & 2.55 & \pm 0.57 & -0.161 & \pm 0.43 & 0.12 & 0.87 & 33.1 & 12.2 & 0.72 \\
\hline Basal & 3.01 & \pm 0.80 & 2.80 & \pm 0.74 & -0.209 & \pm 0.51 & 0.09 & 1.05 & 36.1 & 13.2 & 0.76 \\
\hline Mid & 2.48 & \pm 0.55 & 2.39 & \pm 0.51 & -0.092 & \pm 0.44 & 0.38 & 0.86 & 35.4 & 13.4 & 0.66 \\
\hline Apex & 2.62 & \pm 0.65 & 2.42 & \pm 0.56 & -0.201 & \pm 0.62 & 0.17 & 1.24 & 49.2 & 16.3 & 0.48 \\
\hline$P$ & 0.05 & & 0.07 & & & & & & & & \\
\hline LAD & 2.79 & \pm 0.61 & 2.62 & \pm 0.55 & -0.167 & \pm 0.39 & 0.08 & 0.82 & 30.3 & 10.8 & 0.75 \\
\hline$C x$ & 2.69 & \pm 0.61 & 2.51 & \pm 0.66 & -0.180 & \pm 0.47 & 0.12 & 0.97 & 37.3 & 15.4 & 0.71 \\
\hline RCA & 2.53 & \pm 0.61 & 2.44 & \pm 0.56 & -0.096 & \pm 0.47 & 0.39 & 0.92 & 37.0 & 13.6 & 0.68 \\
\hline$P$ & 0.442 & & 0.629 & & & & & & & & \\
\hline \multicolumn{12}{|l|}{ MPR } \\
\hline Global & 4.24 & \pm 0.69 & 3.73 & \pm 0.76 & -0.214 & \pm 0.76 & 0.25 & 1.46 & 36.4 & 13.3 & 0.46 \\
\hline Basal & 4.53 & \pm 0.90 & 4.27 & \pm 1.00 & -0.262 & \pm 0.89 & 0.23 & 1.78 & 40.4 & 15.3 & 0.55 \\
\hline Mid & 3.84 & \pm 0.70 & 3.73 & \pm 0.66 & -0.113 & \pm 0.83 & 0.57 & 1.60 & 42.4 & 15.5 & 0.26 \\
\hline Apex & 4.42 & \pm 0.90 & 4.12 & \pm 0.76 & -0.293 & \pm 1.04 & 0.25 & 2.06 & 48.1 & 16.0 & 0.30 \\
\hline$p$ & $0.047^{\prime}$ & & 0.130 & & & & & & & & \\
\hline$\angle A D$ & 3.96 & \pm 0.58 & 3.83 & \pm 0.81 & -0.128 & \pm 0.71 & 0.45 & 1.35 & 35.3 & 13.1 & 0.50 \\
\hline$C x$ & 4.47 & \pm 0.93 & 4.14 & \pm 0.93 & -0.325 & \pm 0.86 & 0.13 & 1.76 & 40.8 & 15.7 & 0.54 \\
\hline RCA & 4.06 & \pm 0.76 & 3.91 & \pm 0.66 & -0.150 & \pm 0.89 & 0.49 & 1.72 & 43.3 & 15.7 & 0.23 \\
\hline$p$ & 0.118 & & 0.480 & & & & & & & & \\
\hline
\end{tabular}

$R C$ repeatability coefficient, $R C$ (\%)repeatability coefficient as percentage of the mean, $C V$ within subject coefficient of variation, ICC intraclass correlation coefficient

$\mathrm{p}$ - level of significance using Student's T-test

*=significant at $p<0.05$

excellent agreement for all measurements (Table 4). The highest coefficients of variation were seen in the apical slice.

\section{Discussion}

The main findings of this study are 1) Gadgetron in-line myocardial perfusion mapping by CMR has good short and long-term repeatability, 2) regional assessment of coronary artery territory MBF has good repeatability, 3) MPR is a less reproducible method of assessment than MBF, particularly for regional assessment.

\section{Global perfusion analysis}

We have shown good repeatability of global stress and rest MBF for automated perfusion mapping with CMR, both within one scan and with an interval between scans. Our results are consistent with previous studies, using both invasive and non-invasive estimates of MBF and MPR.

Invasive assessment of coronary flow reserve has a coefficient of variation of $19 \%$ on repeat within minutes [13]. Table 5 contains a summary of the published literature on repeatability of non-invasive MBF measurement. PET repeatability in the published literature has ranged 


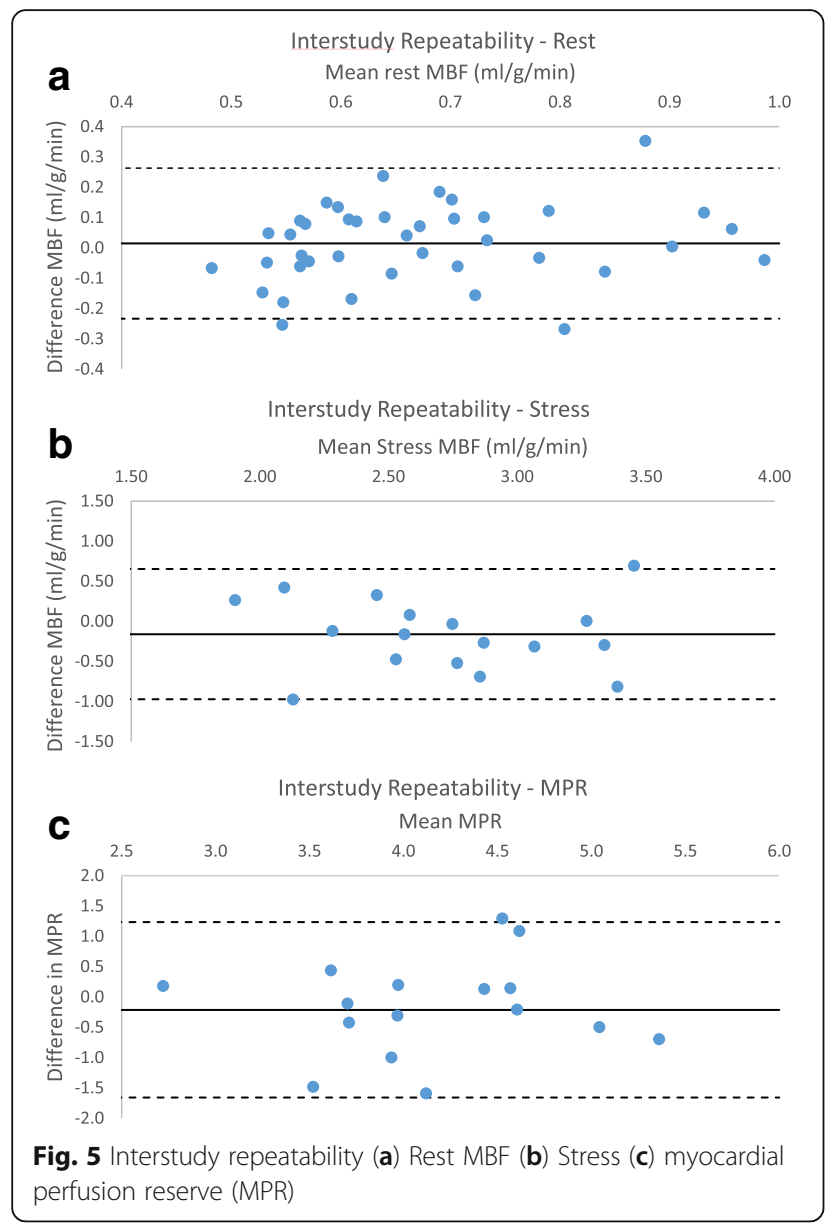

Table 4 Intra and inter-observer MBF reproducibility

\begin{tabular}{|c|c|c|c|c|}
\hline & \multicolumn{2}{|c|}{ Intra-observer } & \multicolumn{2}{|c|}{ Inter-observer } \\
\hline & CV (\%) & ICC & CV (\%) & ICC \\
\hline \multicolumn{5}{|l|}{ Rest } \\
\hline Global & 0.7 & 0.999 & 0.8 & 0.999 \\
\hline Basal & 1.1 & 0.998 & 1.2 & 0.998 \\
\hline Mid & 1.0 & 0.999 & 0.6 & 0.999 \\
\hline Apical & 3.0 & 0.990 & 3.7 & 0.980 \\
\hline LAD & 1.1 & 0.997 & 1.3 & 0.996 \\
\hline$C x$ & 1.4 & 0.997 & 1.3 & 0.995 \\
\hline RCA & 1.8 & 0.996 & 1.8 & 0.991 \\
\hline \multicolumn{5}{|l|}{ Stress } \\
\hline Global & 2.0 & 0.995 & 2.4 & 0.995 \\
\hline Basal & 2.0 & 0.996 & 3.0 & 0.993 \\
\hline Mid & 2.6 & 0.999 & 3.3 & 0.994 \\
\hline Apical & 6.3 & 0.963 & 8.0 & 0.959 \\
\hline LAD & 2.8 & 0.99 & 2.6 & 0.99 \\
\hline$C x$ & 2.5 & 0.992 & 4.3 & 0.986 \\
\hline RCA & 1.9 & 0.992 & 1.9 & 0.993 \\
\hline
\end{tabular}

CV coefficient of variation, ICC intraclass correlation coefficient from RCs of $18-35 \%$ for resting MBF and $18-41 \%$ for stress MBF [14-17]. The largest study of test-retest repeatability in PET to date involved 120 volunteers who underwent serial stress or rest scans. Rest MBF CVs were $10.7 \%$ within test and $21.1 \%$ between tests, and stress MBF CVs were 9.6-10.6 and 19-21\% [18]. As in most of the published literature, short-term repeatability had lower coefficients of variation and repeatability than delayed repeatability. In a recent study to assess the optimal kinetic model for repeatability in PET the best results gave a combined repeatability coefficient of $15.8 \%$ for stress and rest [19].

There are few CMR studies of repeatability. The largest, a subset of the Multi-Ethnic Study of Atherosclerosis (MESA) study, included 30 patients with an interval of almost a year between scans [20], and reported repeatability coefficients of 30 and $41 \%$ for rest and stress MBF respectively, similar to values in PET studies. Another small sample of 10 patients showed good correlation for interstudy repeatability at rest $(r=0.77)$ and stress $(r=0.9)$ and CVs of $23 \%$ at rest and $20 \%$ for stress, in keeping with previous PET literature [21]. Other CMR studies have shown poorer repeatability than PET; a more recent, smaller study of 11 subjects [8] showed repeatability coefficients of 45 and $73 \%$, with coefficients of variation of 20 and $40 \%$ for rest and stress respectively.

Our data for automated in line perfusion CMR mapping fit well within this published data and are comparable to the best results achieved with PET. Compared with most CMR studies, the repeatability in the current study was better for global MBF at both rest and stress, and for both short and long-term repeatability.

\section{Regional assessment}

All coronary territories showed good repeatability for rest and stress MBF. Higher repeatability coefficients and coefficients of variation were seen than for global values, consistent with the limited published literature in CMR and some of the PET literature. These findings likely reflect the smaller volume of myocardium assessed in regional assessment. Early CMR studies of repeatability have included only the mid slice but have shown regional repeatability to be higher than global (RC $28 \%$ vs 21\%) [22]. The PET literature is inconsistent regarding regional vs global repeatability. One PET study examined coronary territory MBF repeatability in 30 patients and reported similar repeatability coefficients to global flow at stress (RC 18\% for global perfusion, $18-24 \%$ for regional perfusion) and at rest (RC 31\% for global and $26-46 \%$ for regional perfusion). A further study of 48 subjects showed comparable values for global perfusion vs regional perfusion (quadrants), with regional rest values of RC $33-41 \%$ and stress $33-38 \%$ vs global values 35 and 
Table 5 Summary of literature on MBF repeatability

\begin{tabular}{|c|c|c|c|c|c|c|c|c|c|c|c|c|c|c|c|}
\hline & & & & Rest & & & & Stress & & & & MPR & & & \\
\hline & Author & Year & $\mathrm{n}$ & T test & $\mathrm{r} / \mathrm{ICC}$ & $\begin{array}{l}\text { RC } \\
(\%)\end{array}$ & $\begin{array}{l}\text { CV } \\
(\%)\end{array}$ & $\mathrm{T}$ test & $r / I C C$ & $\begin{array}{l}\mathrm{RC} \\
(\%)\end{array}$ & $\begin{array}{l}\text { CV } \\
(\%)\end{array}$ & T test & $r / I C C$ & $\begin{array}{l}\text { RC } \\
(\%)\end{array}$ & $\begin{array}{l}\text { CV } \\
(\%)\end{array}$ \\
\hline Imme & te (intrastudy) & & & & & & & & & & & & & & \\
\hline PET & Nitzsche [30] & 1996 & 15 & 0.33 & 0.99 & 33 & & 0.16 & 0.97 & 13 & & & & & \\
\hline & Kaufmann [14] & 1999 & 21 & ns & & 18 & & ns & & 25 & & ns & & 33 & \\
\hline & Wyss [23] & 2003 & 11 & ns & 0.77 & 21 & & & 0.77 & 27 & & ns & 0.74 & 35 & \\
\hline & Schindler [31] & 2007 & 20 & & 0.72 & 29 & & & 0.76 & 20 & & & & & \\
\hline & Manabe [15] & 2009 & 15 & 0.31 & & 22 & & 0.81 & & 27 & & 0.53 & & 37 & \\
\hline & Kitkungvan [18] & 2017 & 120 & 0.93 & & & 11 & 0.74 & & & 10 & & & & \\
\hline & Ocneanu [19] & 2017 & 12 & & & & 21 & & & & 15 & & & & \\
\hline CMR & Keith $^{\mathrm{a}}$ [32] & 2017 & 10 & & & 53 & 13 & & & & & & & & \\
\hline & This study & & & 0.08 & 0.8 & 24 & 8 & 0.41 & 0.76 & 29 & 11 & & & & \\
\hline Delay & (interstudy) & & & & & & & & & & & & & & \\
\hline PET & Nagamachi [16] & 1996 & 30 & ns & 0.63 & 31 & & ns & 0.69 & 18 & & & & 20 & \\
\hline & Schindler [31] & 2007 & 20 & & 0.75 & 30 & & & 0.71 & 23 & & & & & \\
\hline & Sdringola [17] & 2011 & 48 & $p<0.05$ & 0.68 & 35 & & ns & 0.53 & 34 & & ns & 0.47 & 38 & \\
\hline & Johnson [24] & 2015 & 50 & 0.46 & & 41 & & 0.13 & & 34 & & 0.29 & & 34 & \\
\hline & Kitkungvan [33] & 2017 & 19 & 0.13 & & & & 0.94 & & & 17 & 0.26 & & & 20 \\
\hline & Kitkungvan [18] & 2017 & 120 & 0.13 & & & 21 & 0.81 & & & 19 & & & & \\
\hline CMR & Jerosch-Herold [20] & 2008 & 30 & 0.001 & & 30 & & 0.11 & & 41 & & & & & \\
\hline & Larghat $^{\mathrm{a}}[8]$ & 2013 & 11 & 0.2 & & 45 & 20 & 0.61 & & 73 & 40 & 0.11 & & 69 & 35 \\
\hline & Likhite [21] & 2016 & 10 & & 0.77 & & & & & & & & 0.88 & & \\
\hline & Keith $^{\mathrm{a}}$ [32] & 2017 & 10 & & & 61 & 16 & & & & & & & & \\
\hline & This study & & & 0.8 & 0.74 & 32 & 11 & 0.12 & 0.72 & 33 & 12 & 0.25 & 0.44 & 36 & 13 \\
\hline
\end{tabular}

$n s$ not significant ( $p$ value not reported), $r$ Pearson correlation coefficient, $R C$ reproducibility coefficient (\% of mean), CV coefficient of variation

${ }^{a}$ Repeatability data is given for single mid-ventricular slice, all other studies, data is for global myocardium, averaged from multiple slices. Where RC was not published, but sufficient data was provided, this has been calculated using $1.96 *$ SD of difference. Similarly, all RC are given as \% for ease of comparison

34\% [17]. Other PET studies have shown worse repeatability in regional assessment compared to global values. A study of 21 subjects assessing repeatability of ${ }^{15} \mathrm{O}$ PET had regional repeatability coefficients of $22-46 \%$ at rest and $41-59 \%$ at stress vs 18 and $25 \%$ globally [14]. A smaller study of 11 showed regional repeatability coefficients of $38-55 \%$ at rest and $55-70 \%$ at stress vs 21 and $27 \%$ globally [23]. Numerically the regional variability in our study was similar to or lower than in most PET studies. All coronary territories showed good correlation and repeatability with RCs of $32-36 \%$ at rest and $30-37 \%$ at stress.

In addition to coronary territories, we also compared variability between the three acquired slices. At stress the apical slice had the lowest ICC, showing moderate reliability (ICC $=0.48, p=0.02$ ). It also showed the highest inter- and intra-observer variability, with higher coefficients of variation than other regions. This underlines the difficulty in assessment and quantification of the most apical slice, due to the small area of myocardium available for analysis and the larger partial volume effect due to the conical shape of the apex, exacerbated at stress by increased cardiac motion.
MPR assessment

We found that the repeatability of CMR MPR was lower than that of MBF, in particular for the analysis of coronary territories. Previous studies have shown similar values for repeatability of global MPR to resting and stress MBF, with PET values of multiple studies within the range of 33-38\%, in keeping with our results $[14,15,17,23,24]$. A study of 30 subjects showed a repeatability coefficient of $20 \%$ for MPR; however, these results included some studies repeated within the same day, which may account for the lower values. Data on inter-test reproducibility for MPR in CMR is limited, with a single study of 11 subjects showing a RC of $69 \%$, higher than PET data and those from this study [8].

Where regional MPR has been compared to global values for repeatability, some studies have shown markedly worse repeatability. One study showed regional RC of $68-82 \%$ vs $33 \%$ for global values [14] another showed an increase from $35 \%$ for to $67-96 \%$ [23]. Others have shown a small rise or comparable RC in a similar pattern to our data. The largest study, with 48 paired studies showed a RC of $38 \%$ globally with a maximum of $43 \%$ in the lateral wall [17]. 
The lack of significant correlation in repeated results both for apical and mid slices and the RCA territories supports the use of stress MBF rather than MPR for clinical assessment where regional differences are diagnostically important. It is known that stress MBF correlates with the severity of stenosis in CAD [25], therefore the better reliability we have demonstrated for stress MBF compared with MPR would support its use for this important diagnostic decision.

\section{Variation in resting $\mathrm{MBF}$}

We have shown variation in resting MBF on short-term repeat, within the same scan. A trend for rest MBF to decrease with serial measurement was not removed by correction for heart rate. Whilst the level of significance in this difference altered when MBF was corrected for HR, there remained a trend for decrease with sequential repeat (Fig. 3).

Published literature has not shown this decrease in MBF during the same scan. Two studies have shown a decrease in resting $\mathrm{MBF}$ on repeat assessment with a longer interval between imaging. In a study of healthy subjects with no risk factors, a significant decrease of $0.05 \pm 0.13 \mathrm{ml} / \mathrm{g} /$ $\min (p<0.05)$ was seen with a median interval of 22 days between scans [17]. This effect was not present in those with cardiovascular risk factors or in stress perfusion. The MESA study produced a similar finding with a decrease in resting MBF of $0.1 \mathrm{ml} / \mathrm{g} / \mathrm{min}, p=0.001$ over a longer time interval (mean 334 days) [20]. This change was also accompanied by a significant change in heart rate, which was proposed to account for the drop.

Although some of these results differ from those in our study and the intervals between scans were different from in our study, they do provide more evidence of the susceptibility of rest MBF to change and physiological variation. Our study is the first to report more than 2 repeated measurements within one scanning session. The decrease seen within our study may be caused by an element of anxiety present at the beginning of the CMR scan in the subjects examined. In addition to a rise in heart rate and blood pressure, stress is associated with vasodilation of the coronary microvasculature in healthy volunteers [26]. Following correction for heart rate, the decrease in MBF remained, suggesting that vasodilation rather than cardiac work is the cause of the higher values on the first scans. This assumption is supported by the lack of difference between sequential stress perfusion, where maximal vasodilatation is induced so that differences in anxiety have no additional impact. The absence of significant difference in sequential stress scans also supports that this change is likely to be physiological rather than an effect of residual Gd from the previous series. The lack of significant difference between the second perfusion acquisition and subsequent assessment is reassuring clinically, as this would imply that stress followed by rest perfusion assessment, the most commonly used protocol, would produce repeatable values for both rest and stress.

\section{In-line perfusion mapping}

The development of Gadgetron automated inline perfusion mapping overcomes one of the main previous limitations of quantitative perfusion CMR by removing the previously time-consuming analysis and the need for specialist knowledge. We have shown that this technique provides measurements of MBF with a repeatability that is comparable to the reference method PET and that is at least as good as previous, manual, CMR analysis methods. A recent study has shown that CMR perfusion mapping yields similar MBF values at rest and vasodilator stress as ammonia PET in patients with stable CAD [10]. Assessment using perfusion maps allows objective assessment of MBF, providing simpler and faster analysis and may have clinical advantages of detecting disease with global decrease in MBF such as microvascular and multivessel disease.

\section{Study limitations}

Our data are influenced by physiological variation as well as variation within the model and analysis. While we aimed to minimise physiological variation as much as possible, some effects may not have been controlled for. Caffeine has been demonstrated to affect coronary vasomotor tone at rest [27] and adenosine stress perfusion CMR [28]. Although we advised our volunteers to avoid caffeine for $24 \mathrm{~h}$ prior to the scan, previous studies have demonstrated that up to $20 \%$ may still have detectable caffeine levels [29]. In addition, dosing of adenosine was determined clinically according to symptoms and response rather than a direct repeat from the previous scan. This would mimic clinical practice, and any difference in doses required may result in physiological variants in response. We can be confident that adequate stress was achieved; having seen appropriate increases in HR and RPP together with symptomatic response, however, the degree of hyperaemia may vary from maximal and account for the increased variation seen in stress MBF. All studies were performed using a FLASH perfusion sequence at $3 \mathrm{~T}$, results for MBF may vary using other sequences or field strengths.

\section{Conclusion}

Quantitative perfusion CMR using automated perfusion mapping achieves estimates of MBF and MPR with repeatability similar to the reference standard method PET. In this study rest and stress MBF, rather than MPR were a more reproducible assessment, particularly in regional analysis. The degree of physiological 
variation emphasises the importance in establishing normal ranges to allow for accurate diagnostic use.

\section{Additional files}

Additional file 1: Figure S1. Correlation by slice (A) rest (B) stress,

Trend line represents line of perfect fit. (DOCX $119 \mathrm{~kb}$ )

Additional file 2: Figure S2. Correlation by coronary territory (A) rest (B) stress. Trend line represents line of perfect fit. (DOCX $116 \mathrm{~kb}$ )

\section{Abbreviations}

AHA: American Heart Association; AIF: arterial input function; ANOVA: analysis of variance; BP: blood pressure; BTEX: blood tissue exchange; CAD: coronary artery disease; CFR: coronary flow reserve; CMR: cardiovascular magnetic resonance; CV: coefficient of variation; CX: circumflex; ECG: Electrocardiogram; FFR: fractional flow reserve; Gd: gadolinium; HR: heart rate; ICC: intraclass correlation coefficient; IQR: interquartile range; LAD: left anterior descending; MBF: myocardial blood flow; MOCO: motion corrected; MPR: myocardial perfusion reserve; PD: Proton density; PET: Positron emission tomography; RC: repeatability coefficient; RCA: right coronary artery; RPP: rate pressure product: SD: standard deviation; TE: Echo time; TR: Repetition time;

TS: Saturation recovery time

\section{Acknowledgements}

We thank David Shelley, Gavin Bainbridge, Petra Biisterveld, Fiona Richards and Hannah Newman for their help in recruiting and scanning patients and the organisation of running this study.

\section{Funding}

This work was supported by British Heart Foundation grants CH/16/2/32089 and $R G / 16 / 1 / 32092$ to Sven Plein.

\section{Availability of data and materials}

The datasets used and/or analysed during the current study are available from the corresponding author on reasonable request.

\section{Authors' contributions}

LB: Conception and design, recruitment, supervision of scans, analysis and interpretation of data, drafting of manuscript. SO: Supervision of scans, analysis and interpretation of data, critical \& intellectual revision of manuscript. DB: analysis and interpretation of data, critical \& intellectual revision of manuscript. KJ: Recruitment, supervision of scans, critical \& intellectual revision of manuscript. GF: Critical \& intellectual revision of manuscript. JF: Critical \& intellectual revision of manuscript. PG: Critical \& intellectual revision of manuscript. PC: Critical \& intellectual revision of manuscript. KK: Critical \& intellectual revision of manuscript. ED: Critical \& intellectual revision of manuscript. PS: Critical \& intellectual revision of manuscript. HX: Conception and design, processing and interpretation of CMR data, critical \& intellectual revision of manuscript. JG: Critical \& intellectual revision of manuscript. JM: Critical \& intellectual revision of manuscript. PK: Conception and design, processing and interpretation of CMR data, critical \& intellectual revision of manuscript. SP: Conception and design, interpretation of data and drafting of manuscript. All authors read and approved the final manuscript.

\section{Ethics approval and consent to participate}

The study was performed in accordance with the Declaration of Helsinki (October 2000), with all volunteers providing informed written consent. Ethical approval was given by NRES Committee Yorkshire and the Humber Leeds West, Ref 12/YH/0551.

\section{Competing interests}

The authors declare that they have no competing interests.

\section{Publisher's Note}

Springer Nature remains neutral with regard to jurisdictional claims in published maps and institutional affiliations.

\section{Author details}

'Multidisciplinary Cardiovascular Research Centre (MCRC) \& Leeds Institute of Cardiovascular and Metabolic Medicine, University of Leeds, Clarendon Way, Leeds LS2 9JT, UK. ${ }^{2}$ Medical Physics and Engineering, Leeds Teaching Hospitals NHS Trust, Leeds LS1 3EX, UK. ${ }^{3}$ Barts Heart Centre, The Cardiovascular Magnetic Resonance Imaging Unit and The Inherited Cardiovascular Diseases Unit, St Bartholomew's Hospital, West Smithfield, London, UK. ${ }^{4}$ National Heart, Lung, and Blood Institute, National Institutes of Health, DHHS, Bethesda, MD, USA.

Received: 1 December 2017 Accepted: 23 May 2018 Published online: 09 July 2018

\section{References}

1. Tonino PAL, Fearon WF, De Bruyne B, Oldroyd KG, Leesar MA, Ver Lee PN, et al. Angiographic versus functional severity of coronary artery Stenoses in the FAME study fractional flow reserve versus angiography in multivessel evaluation. J Am Coll Cardiol. 2010;55:2816-21.

2. Fischer JJ, Samady H, McPherson JA, Sarembock IJ, Powers ER, Gimple LW, et al. Comparison between visual assessment and quantitative angiography versus fractional flow reserve for native coronary narrowings of moderate severity. Am J Cardiol. 2002:90:210-5.

3. Hamilos M, Muller O, Cuisset T, Ntalianis A, Chlouverakis G, Sarno G, et al. Long-term clinical outcome after fractional flow reserve-guided treatment in patients with angiographically equivocal left main coronary artery stenosis. Circulation. 2009:120:1505-12.

4. Lockie T, Ishida M, Perera D, Chiribiri A, De Silva K, Kozerke S, et al. Highresolution magnetic resonance myocardial perfusion imaging at 3.0-tesla to detect hemodynamically significant coronary stenoses as determined by fractional flow reserve. J Am Coll Cardiol. 2010;57:70-5. https://doi.org/10. 1016/j.jacc.2010.09.019.

5. Christian TF, Rettmann DW, Aletras AH, Liao SL, Taylor JL, Balaban RS, et al. Absolute myocardial perfusion in canines measured by using dual-bolus first-pass MR imaging. Radiology. 2004;232:677-84. https://doi.org/10.1148/ radiol.2323030573

6. Wilke $N$, Jerosch-Herold M, Wang Y, Huang Y, Christensen BV, Stillman AE, et al. Myocardial perfusion reserve: assessment with multisection, quantitative, first-pass MR imaging. Radiology. 1997;204:373-84. https://doi. org/10.1148/radiology.204.2.9240523.

7. Jerosch-Herold M, Swingen C, Seethamraju RT. Myocardial blood flow quantification with MRI by model-independent deconvolution. Med Phys. 2002;29:886-97.

8. Larghat AM, Maredia N, Biglands J, Greenwood JP, Ball SG, Jerosch-Herold $M$, et al. Reproducibility of first-pass cardiovascular magnetic resonance myocardial perfusion. J Magn Reson Imaging. 2013;37:865-74.

9. Kellman P, Hansen MS, Nielles-Vallespin S, Nickander J, Themudo R, Ugander $\mathrm{M}$, et al. Myocardial perfusion cardiovascular magnetic resonance: optimized dual sequence and reconstruction for quantification. J Cardiovasc Magn Reson. 2017;19:43. https://doi.org/10.1186/s12968-017-0355-5.

10. Engblom H, Xue H, Akil S, Carlsson M, Hindorf C, Oddstig J, et al. Fully quantitative cardiovascular magnetic resonance myocardial perfusion ready for clinical use: a comparison between cardiovascular magnetic resonance imaging and positron emission tomography. J Cardiovasc Magn Reson. 2017:19:78. https://doi.org/10.1186/s12968-017-0388-9.

11. Cerqueira MD, Weissman NJ, Dilsizian V, Jacobs AK, Kaul S, Laskey WK, et al. Standardized myocardial segmentation and nomenclature for tomographic imaging of the heart. A statement for healthcare professionals from the cardiac imaging Committee of the Council on clinical cardiology of the American Heart Association. Circulation. 2002;105:539-42. https://doi.org/10. 1161/01.CIR.0000060923.07573.F2.

12. Coefficient of variation from duplicate measurements. https:/www.medcalc org/manual/cvfromduplicates.php. Accessed 14 Mar 2018.

13. Ng MKC, Yeung AC, Fearon WF. Invasive assessment of the coronary microcirculation: superior reproducibility and less hemodynamic dependence of index of microcirculatory resistance compared with coronary flow reserve. Circulation. 2006;113:2054-61.

14. Kaufmann PA, Gnecchi-Ruscone T, Yap JT, Rimoldi O, Camici PG. Assessment of the reproducibility of baseline and hyperemic myocardial blood flow measurements with 150-labeled water and PET. J Nucl Med. 1999:40:1848-56. 
15. Manabe O, Yoshinaga K, Katoh C, Naya M, Dekemp RA, Tamaki N. Repeatability of rest and hyperemic myocardial blood flow measurements with 82 Rb dynamic PET. J Nucl Med. 2009;50:68-71.

16. Nagamachi S, Czernin J, Kim A, Sun K, Bottcher M, Phelps ME, et al. Reproducibility of measurements of regional resting and hyperemic myocardial blood flow assessed with PET. J Nucl Med. 1996;37:1626-31.

17. Sdringola S, Johnson NP, Kirkeeide RL, Cid E, Gould KL. Impact of unexpected factors on quantitative myocardial perfusion and coronary flow reserve in young, asymptomatic volunteers. JACC Cardiovasc Imaging. 2011;4:402-12.

18. Kitkungvan D, Johnson NP, Roby AE, Patel MB, Kirkeeide R, Gould KL. Routine clinical quantitative rest stress myocardial perfusion for managing coronary artery disease: Clinical Relevance of Test-Retest Variability. JACC Cardiovasc Imaging. 2017;

19. Ocneanu AF, DeKemp RA, Renaud JM, Adler A, Beanlands RSB, Klein R. Optimally repeatable kinetic model variant for myocardial blood flow measurements with 82Rb PET. Comput Math Methods Med. 2017;

20. Jerosch-Herold M, Vazquez G, Wang L, Jacobs DRJ, Folsom AR. Variability of myocardial blood flow measurements by magnetic resonance imaging in the multi-ethnic study of atherosclerosis. Investig Radiol. 2008:43:155-61.

21. Likhite D, Suksaranjit P, Adluru G, Hu N, Weng C, Kholmovski E, et al. Interstudy repeatability of self-gated quantitative myocardial perfusion MRI. J Magn Reson Imaging. 2016:43:1369-78.

22. Elkington AG, Gatehouse PD, Ablitt NA, Yang G-Z, Firmin DN, Pennell DJ. Interstudy reproducibility of quantitative perfusion cardiovascular magnetic resonance. J Cardiovasc Magn Reson. 2005;7:815-22.

23. Wyss CA, Koepfli P, Mikolajiczyk K, Burger C, Von Schulthess GK, Kaufmann PA Bicycle exercise stress in PET for assessment of coronary flow reserve: repeatability and comparison with adenosine stress. J Nucl Med. 2003;44:146-54.

24. Johnson NP, Gould KL. Regadenoson versus dipyridamole hyperemia for cardiac PET imaging. JACC Cardiovasc Imaging. 2015;8:438-47.

25. Uren NG, Melin JA, de Bruyne B, Wijns W, Baudhuin T, Camici PG. Relation between myocardial blood flow and the severity of coronary artery stenosis. N Engl J Med. 1994;330:1782-8.

26. Dakak N, Quyyumi AA, Eisenhofer G, Goldstein DS, lii RC. Sympathetically mediated effects of mental stress on the cardiac microcirculation of patients with Coranary artery disease. Am J Cardiol. 1995;76:125-30.

27. Bottcher M, Czernin J, Sun K, Phelps ME, Schelbert HR. Effect of caffeine on myocardial blood flow at rest and during pharmacological vasodilation. J Nucl Med. 1995;36:2016-21.

28. Oudkerk M, Kuijpers D, Prakken NH, Vliegenthart R, van Dijkman M, Pvdijkman PR, van der Harst pvanderharst bronovonl P, et al. Caffeine intake inverts the effect of adenosine on myocardial perfusion during stress as measured by T1 mapping. Int J Cardiovasc Imaging. 2016;32:1545-53. https://doi.org/10.1007/s10554-016-0949-2

29. Banko LT, Haq SA, Rainaldi DA, Klem I, Siegler J, Fogel J, et al. Incidence of caffeine in serum of patients undergoing dipyridamole myocardial perfusion stress test by an intensive versus routine caffeine history screening. AJC. 2010; 105:1474-9. https://doi.org/10.1016/i.amjcard.2009.12.072

30. Nitzsche EU, Choi Y, Czernin J, Hoh CK, Huang S, Schelbert HR. Noninvasive Quantification of Myocardial Blood Flow in Humans A Direct Comparison of the [ N]Ammonia and the [ O]Water Techniques. Circ Cardiovasc Imaging. 1996;93:2000-6.

31. Schindler TH, Zhang XL, Prior JO, Cadenas J, Dahlbom M, Sayre J, et al. Assessment of intra- and interobserver reproducibility of rest and cold pressor test-stimulated myocardial blood flow with $13 \mathrm{~N}$-ammonia and PET. Eur J Nucl Med Mol Imaging. 2007;34:1178-88.

32. Keith GA, Rodgers CT, Chappell MA, Robson MD. A Look-Locker Acquisition Scheme for Quantitative Myocardial Perfusion Imaging with FAIR Arterial Spin Labeling in Humans at 3 Tesla. Magn Reson Med. 2017;78:541-9.

33. Kitkungvan D, Lai D, Zhu H, Roby AE, Patel MB, Kirkeeide R, et al. Optimal Adenosine Stress for Maximum Stress Perfusion, Coronary Flow Reserve, and Pixel Distribution of Coronary Flow Capacity by Kolmogorov-Smirnov Analysis. Circ Cardiovasc Imaging. 2017. https://doi.org/10.1161/ CIRCIMAGING.116.005650.

\section{Ready to submit your research? Choose BMC and benefit from:}

- fast, convenient online submission

- thorough peer review by experienced researchers in your field

- rapid publication on acceptance

- support for research data, including large and complex data types

- gold Open Access which fosters wider collaboration and increased citations

- maximum visibility for your research: over $100 \mathrm{M}$ website views per year

At BMC, research is always in progress.

Learn more biomedcentral.com/submissions 